\title{
Hemorrhage as a Complication of Percutaneous Liver Biopsy
}

\author{
Darren van Beek, MD ${ }^{1}$ Brian Funaki, MD ${ }^{1}$ \\ ${ }^{1}$ Section of Vascular and Interventional Radiology, University of \\ Chicago Medical Center, Chicago, Illinois \\ Semin Intervent Radiol 2013;30:413-416
}

\begin{abstract}
Address for correspondence Brian Funaki, MD, Section of Vascular and Interventional Radiology, University of Chicago Medical Center, 5840 S. Maryland Avenue, MC 2026, Chicago, IL 60637 (e-mail: bfunaki@radiology.bsd.uchicago.edu).
\end{abstract}

Percutaneous biopsies of the abdominal solid organs, particularly the liver, are among the most well-established and frequently performed interventional procedures. While offering lower morbidity than open surgical biopsies, these procedures are not risk free and postbiopsy hemorrhage is one of the most frequently encountered complications. Interventionists must therefore be well versed in preprocedure patient optimization, postbiopsy evaluation, and management of hemorrhagic complications.

\section{Case Report}

A 70-year-old woman with a history of infiltrating ductal carcinoma of the breast was referred to the Vascular and Interventional Radiology Section for biopsy of a new liver lesion suspicious for a hepatic metastasis. Multiple hypodense lesions were present, the largest of which had a subcapsular location in the right hepatic lobe (-Fig. 1).

Before the biopsy, the patient's hematologic serologies were within accepted limits (international normalized ratio $(\mathrm{INR})=1.2$, platelets $=102,000 / \mu \mathrm{L}, \mathrm{Hgb}=12.5 \mathrm{~g} / \mathrm{dL})$. After an initial sonographic survey, the large right lobe lesion was selected for biopsy. The biopsy was performed under continuous ultrasonographic guidance using co-axial technique (-Fig. 2). A fine needle aspiration was performed using a 20 -g needle and then three 20 -g core biopsies were obtained using an automatic biopsy device. The patient tolerated the procedure well and was transferred to the postoperative holding area. Approximately 1 hour after the biopsy, the patient became somnolent, tachycardic ( 146 beats per minute (BPM)), and hypotensive (76/41 $\mathrm{mm} \mathrm{Hg}$ ). The patient was intubated for airway protection and a stat laboratory analysis showed a hemoglobin level of $5.9 \mathrm{~g} / \mathrm{dL}$.

Resuscitation was performed with $1 \mathrm{~L}$ of $0.9 \%$ normal saline, 9 units of packed red blood cells, 3 units of platelets, and 2 units of fresh frozen plasma. A computed tomographic (CT) scan of the abdomen and pelvis performed with and without intravenous contrast showed the previously identi- fied subcapsular right hepatic lobe lesion with new biopsy defects. There was an associated large perihepatic hematoma, high-density free fluid in the abdomen, and active extravasation from the biopsy site (-Fig. $\mathbf{3}$ ).

The patient underwent emergent angiography with intent to embolize the active hemorrhage. Right common femoral artery access was obtained using a 5-Fr coaxial micropuncture kit (Cook Medical, Bloomington, IN). The celiac axis was catheterized using a 5-Fr RC1 catheter (Cook Medical). Angiograms of the celiac axis, right hepatic, and left hepatic arteries were performed that showed variant anatomy (-Fig. 4) with the right hepatic artery arising directly from the celiac axis and active extravasation from multiple peripheral branches of the right hepatic artery. Superselective embolization was unable to be performed due to vasospasm. Embolization of the right hepatic artery was performed using a Gelfoam (Pfizer, New York, NY) slurry until pruning occurred in distal branches. Repeat right hepatic artery angiogram showed hemostasis. After the procedure, the patient remained hemodynamically stable without need for further blood product transfusion. Pathology results confirmed the lesion to be a breast cancer metastasis.

\section{Discussion}

Percutaneous abdominal solid organ biopsies are among the most commonly performed interventional procedures. While possessing lower morbidity and mortality than open surgical biopsies, they possess their own set of risks. ${ }^{1}$ Ultrasound is the preferred modality for image guidance in percutaneous liver biopsies in most patients, as it offers real-time, multiplanar localization of both the needle and the target lesion. Alternatively, CT guidance may be preferred when there is a large skin-to-target distance owing either to the lesions location within the liver or the patient's body habitus.

A needle path devoid of intervening bowel and significant biliary structures is required for biopsy. While technically feasible to perform liver biopsies by traversing the pleural space and
Issue Theme Women's Health and Interventional Radiology; Guest Editors, Kimi L. Kondo, DO, and Laura Findeiss, MD, FSIR
Copyright (c) 2013 by Thieme Medical Publishers, Inc., 333 Seventh Avenue, New York, NY 10001, USA. Tel: +1(212) 584-4662.
DOI http://dx.doi.org/ 10.1055/s-0033-1359737. ISSN 0739-9529. 


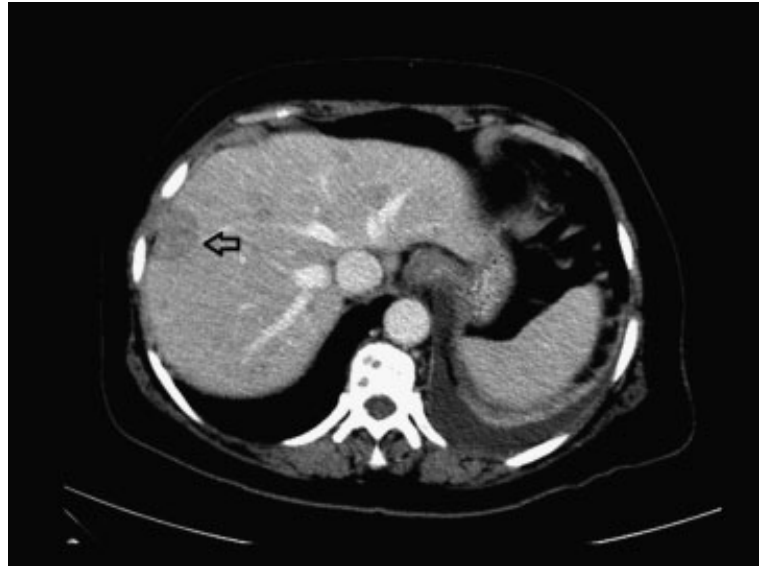

Figure 1 Contrast-enhanced CT through the liver showing multiple hypodense parenchymal lesions. The largest lesion (subcapsular, right hepatic lobe-open arrow) was chosen for biopsy, which confirmed metastatic breast cancer.

unaerated lung, this is discouraged due to both the risk of pneumothorax and the potential for seeding of the pleural space with either malignant or infectious debris (depending on the source lesion). Beyond the above considerations, the primary concerns when evaluating a patient for potential percutaneous biopsy are hemorrhagic and vascular complications. Before biopsy, patients should have a coagulation profile performed. The consensus Society of Interventional Radiology guidelines for percutaneous liver biopsy recommend safe thresholds of a partial thromboplastin time (PTT) $<1.5$ times the upper limit of normal, INR $<1.5$, and platelets $>50,000 / \mu L^{2}$

The choice of biopsy needle size is an exercise in balancing the stiffness and tissue yield offered by larger needles with a mildly increased risk of postbiopsy hemorrhage attributed to larger needles. In most cases, a 20-gauge biopsy needle or automatic biopsy device provides sufficient control and tissue yield while maintaining an acceptable bleeding risk.

In 1985, Solbiati et al recommended using a needle path that traverses 1 to $2 \mathrm{~cm}$ of normal hepatic parenchyma before entering the target lesion when performing percutaneous

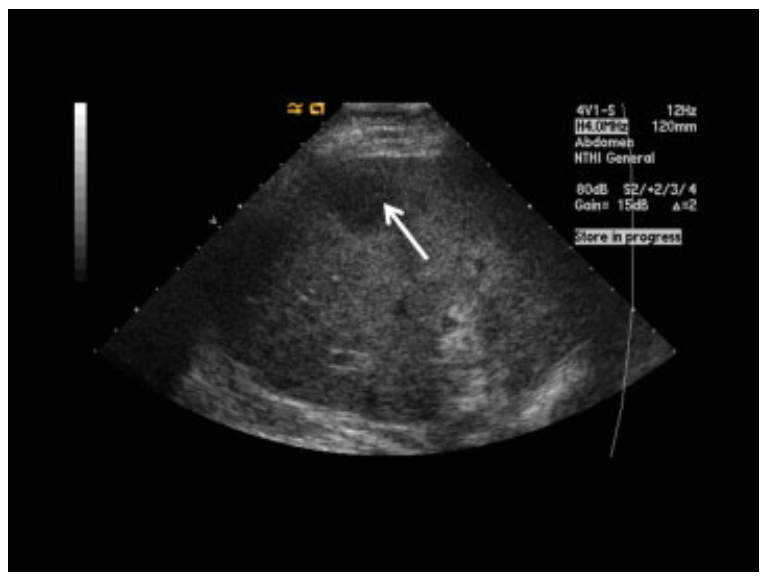

Figure 2 Grayscale ultrasound image of the target lesion (arrow) before percutaneous biopsy.

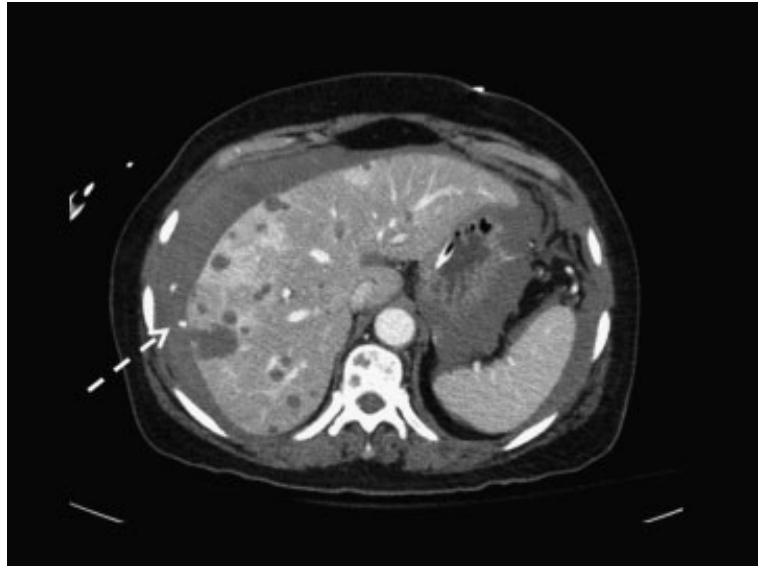

Figure 3 Postbiopsy CT scan through the liver redemonstrates the target lesion. There is a new, large-volume perihepatic and free abdominal fluid collection. A smaller hyperdense focus adjacent to the biopsy site indicates active extravasation (arrow).

liver biopsies. ${ }^{3}$ This allows for a tamponade effect by the relatively dense surrounding normal hepatic parenchyma to minimize bleeding from the biopsy site. To the authors' knowledge, no rigorous studies have been performed to validate this recommendation, but it has rather become standard practice through a combination of an intuitive underlying principal and operator experience. In this particular case, an insufficient length of normal parenchyma likely contributed to the postbiopsy hemorrhage from the highly vascular metastasis.

Single-needle, dual-needle, and coaxial techniques for liver biopsy are all well established in the literature and in practice. The choice of technique is typically based on performing clinician preference with deeper and more technically challenging biopsies potentially favoring either the dualneedle or coaxial techniques.

Postbiopsy is standard practice at our institution to repeat a sonographic survey of the biopsy area to evaluate for a developing subcapsular or pericapsular hematoma. Similarly, if the biopsy is performed under CT guidance, a postbiopsy scan through the entire liver should be performed. Once the procedure is finished, the patient should be observed for 2 to 4 hours with frequent monitoring of their vital signs and clinical stability. In the absence of complicating factors, percutaneous liver biopsy can be safely performed on an outpatient basis with the patient being discharged after the observation period.

Clinical evidence of hypovolemia in the postbiopsy setting is presumed to reflect hemorrhage from the biopsy site until proven otherwise. As the injury is essentially a penetrating trauma, basic trauma protocol should be initiated including volume resuscitation with crystalloid fluids and a basic laboratory analysis including at least a hemoglobin count and coagulation parameters. ${ }^{4}$ If the patient is clinically unstable, immediate surgical consultation and operative management is imperative. In most cases, the rate of hemorrhage is slow enough that patient can be stabilized with volume resuscitation and further diagnostic workup performed. If 

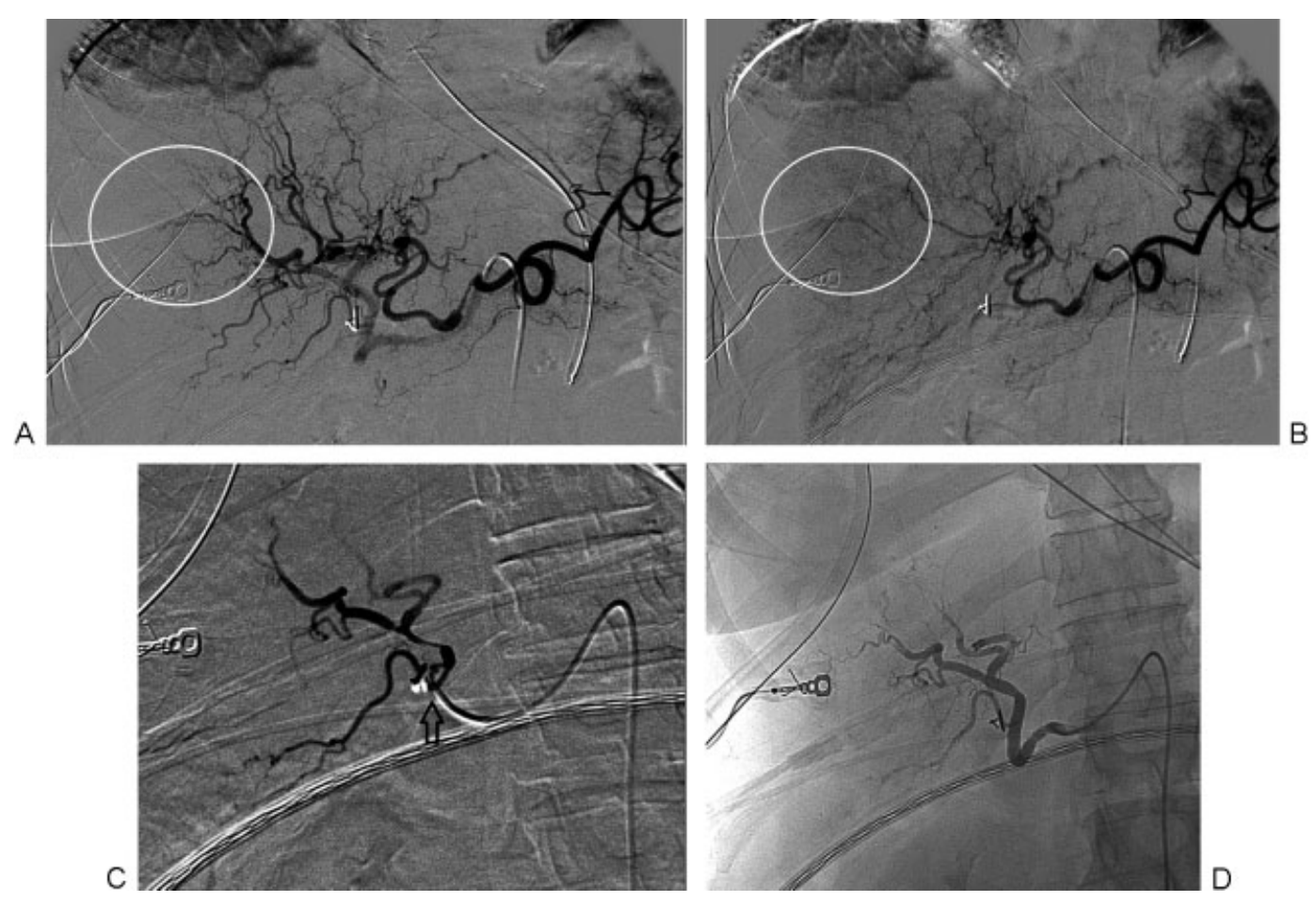

Figure 4 (A) Digital subtraction angiogram of the celiac axis showing active extravasation of contrast from peripheral branches of the right hepatic artery (encircled). (B) Delayed image from the right hepatic arteriogram shows persistence of the extravasated contrast (encircled). (C) Postembolization right hepatic arteriogram shows pruning of the peripheral vessel branches and no further contrast extravasation. The catheter position (arrow) shows the point at which the embolization was performed. (D) Unsubtracted image from postembolization right hepatic arteriogram redemonstrating pruning of the peripheral vessel branches and no further contrast extravasation.

stable, imaging with CT can be considered. Evidence of continued hemorrhage based either on active extravasation on the CT or an ongoing need for blood transfusions merits angiographic evaluation and possible embolization.

Clinically significant hemorrhage from the liver is almost exclusively arterial in origin. In contrast to most intra-abdominal organs, arterial embolization in the liver is typically well tolerated due to the liver's dual blood supply. Exceptions to this rule include chronic liver disease, where the patient may not tolerate further loss of functional parenchyma, and portal venous thrombosis, where the liver's dual blood supply is compromised. Special care must also be taken in the posttransplant liver where the vascular supply is more tenuous and the risk of ischemic biliary ductal complications is higher.

After obtaining arterial access but before embolization, a superior mesenteric artery angiogram should be obtained through the venous phase to evaluate the patency of the portal vein and thus confirming a dual blood supply to the liver. If the portal venous supply is compromised, a more selective embolization should be performed because there is potentially an increased risk of ischemic complications. In the current case, the CT scan obtained immediately before angiography showed a widely patent portal vein obviating the need for this angiographic step.

Embolization can be performed with either Gelfoam or microcoils. A Gelfoam slurry enables rapid embolization of multiple bleeding sites from a more proximal artery. Gelfoam embolization of the proper hepatic artery can be performed; however, the embolization should be performed distal to the origin of the cystic artery when possible. The gallbladder lacks the dual blood supply of the liver and accidental embolization of the cystic artery can result in gallbladder ischemia. Postembolization angiography should demonstrate stasis of contrast within target vessels for at least three to five cardiac cycles and no further active extravasation.

If the rate of bleeding is very brisk and focal, microcoil embolization may be more effective. When using microcoils, the embolization should be as selective and peripheral as possible to minimize the affected area and thus decreases the risk of postembolization infarction. Coil embolization is also preferred for the management of arteriovenous and arteriobiliary fistulae. In cases were hemostasis is difficult to obtain, microcoils and Gelfoam can be used in combination to create a more robust embolization.

Ultimately, the greatest morbidity and mortality from postbiopsy hemorrhages occur when there is a delay in diagnosis or a failure to pursue aggressive management. This emphasizes the need for close postprocedure observation and physician familiarity with the management of hemorrhagic complications.

\section{Financial Disclosures}

The authors have no financial or professional relations to disclose. 


\section{References}

1 Smith EH. Complications of percutaneous abdominal fine-needle biopsy. Review. Radiology 1991;178(1):253-258

2 Patel IJ, Davidson JC, Nikolic B, et al; Standards of Practice Committee, with Cardiovascular and Interventional Radiological Society of Europe (CIRSE) Endorsement. Consensus guidelines for periprocedural management of coagulation status and hemostasis risk in percutaneous image-guided interventions. J Vasc Interv Radiol 2012;23(6):727-736

3 Solbiati L, Livraghi T, De Pra L, Ierace T, Masciadri N, Ravetto C. Fine-needle biopsy of hepatic hemangioma with sonographic guidance. AJR Am J Roentgenol 1985;144(3):471-474

4 Taourel P, Vernhet H, Suau A, Granier C, Lopez FM, Aufort S. Vascular emergencies in liver trauma. Eur J Radiol 2007;64(1):73-82 